\title{
Review of: "Spatial Distribution of Stunting and Its Associated Factors Among Under-five Children in Ethiopia: Spatial and Multilevel Analysis"
}

\author{
Nebyu Daniel Amaha ${ }^{1}$
}

1 Mekelle University

Potential competing interests: The author(s) declared that no potential competing interests exist.

1. Page 1: Title; The authors need to specify that this is based on the 2019 mini Ethiopian Demographic Health Survey (EDHS) in the title, because similar studies were carried out using previous EDHS data. 2. Page 3: Introduction; 2nd paragraph: The authors mentioned that there are national policies to reduce stunting. What is the target reduction of stunting outlined in the Ethiopian National Nutrition Program Phase 1 and Phase 2.

3. Page 3: Introduction; 3rd paragraph: "Despite these interventions, the stunting rate among children in Ethiopia is high..." It is not clear what 'these' exact interventions are. Therefore, the authors need to specify what interventions were implemented and reason out why the interventions did not succeed in reducing stunting levels. For example, were the right number and scale of interventions selected? How was their implementation measured? What were the strengths and weaknesses of the interventions etc 4. Page 3: Introduction; 3rd paragraph: The authors did not put a reference to the previous studies which did they claim as 'old'. Furthermore, they need to specify the finding from these similar spatial and multilevel studies. The paper could benefit from a thorough literature review of previous similar studies carried out in Ethiopia.

5. Page 3: Methodology, sampling procedure: The authors need to put a reference of the sampling procedure used.

6. Page 4: Methodology, study variables: The authors have selected certain individual and community-level factors as predictor variables. However, it is not clear how they chose these specific variables and not others. If their decision was based on previous findings then they clearly need to mention it along with the references upon which their choices were based.

7. Page 4: Methodology, data management and analysis: A simple sample weight was carried out, which is required when all we need is indicator estimate. However, the DHS recommends using complex sample design when standard errors, confidence intervals or significance testing is required. In addition to using sample weight, the authors need to take complex sample design into consideration because their paper makes estimates of the above mentioned variables. For example in STATA the authors can use svyset and svy commands to apply complex sample design.

8. Page 13: The discussion of the spatial sat scan analysis on page 13 is incomplete and very shallow. For 
example, is the acidity in Tigray, Amhara and Afar regions higher than the rest of the country? The authors need to insert citations regarding their theory soil acidity in these regions and lower crop production which would lead to higher levels of stunting in these regions

9. Discussion: This paper could be improved significantly by making comparisons with previous similar studies. At this stage the authors are simply repeating their results in the discussion section. For example, this paper does not mention the numerical findings of previous studies and how their present findings differ from the previous studies 10. Page 14: Discussion; 2nd paragraph: More explanation on "preferential feeding" and how boys are expected to grow faster and still have higher incidence of stunting than girls

11. Table 3: The resuls show that stunting was highest among infants aged 24-35 months. This needs to be included in the discussion with citations from previous findings. The authors mentioned on page 14, 1st paragraph as age the child gets older then the risk of stunting increases. Therefore, wouldn't we expect the stunting to be highest among children aged 48-59 months?

12. Page 15: Strenghts and limitations: The authors mention that they could not include dietary factors, however, the DHS does collect data regarding infant and childhood feeding practices, for example dietary diversity, breastfeeding etc.

13. Gambella region has lower stunting than the capital and the authors have not discussed this finding.

14. Table 3: The exact $p$-value is more preferrable method of reporting than categorical $p$-value. 